\title{
Micro Needle Array-Doxorubicin
}

National Cancer Institute

\section{Source}

National Cancer Institute. Micro Needle Array-Doxorubicin. NCI Thesaurus. Code C156665.

A formulation composed of dissolvable small, adhesive-like patches composed of a biocompatible material which is coated with the anthracycline antibiotic doxorubicin, with potential antineoplastic and immunomodulating activities. Upon cutaneous administration of the microneedle-array-doxorubicin, the microneedles degrade once inserted into the skin and doxorubicin is released from the dissolvable microneedle array delivery device directly into the tumor microenvironment (TME). Doxorubicin is taken up by tumor cells and intercalates into DNA and interferes with topoisomerase II activity. This inhibits DNA replication and RNA synthesis, leading to tumor cell growth inhibition and apoptosis. This agent also interacts with cell membrane lipids causing lipid peroxidation. In addition, doxorubicin induces innate, adaptive, and tumor-specific effector and memory immune responses, thereby further killing the tumor cells. Delivery of doxorubicin using the microneedle array delivery system allows direct and specific administration of doxorubicin to the TME which may improve drug concentration into tumor cells and may reduce systemic toxicity, compared to the administration of systemic doxorubicin alone. 